\title{
0412 DEVELOPMENTAL INFLUENCES ON CHILDREN'S PEDESTRIAN ROUTE SELECTION
}

B Barton, T Ulrich* Correspondence: Department of Psychology and Communication Studies, University of Idaho, Student Health 003, PO Box 443043, Moscow, ID, 83844-3043, USA

\subsection{6/ip.2010.029215.412}

The aim of this research was to replicate and extend previous work by examining cognitive developmental predictors of children's selection of safe routes through pedestrian environments. Schwebel and Barton (2007) found child sex, age and temperamental inhibitory control to be significant predictors of selecting risky pedestrian routes. Although younger age significantly predicted selection of riskier routes, the question of what developmental (or age-related) characteristics are responsible for selecting riskier routes remained unanswered.

We first assessed children's cognitive speed and efficiency, and visual search skills in a series of laboratory tasks. Next, we measured children's route selections during a series of crossings using a wind-up toy on a scale indoor table-top model of a real street. 65 children ages 5-9 participated; 44\% male.

Exploratory Pearson correlations indicated boys, younger children, those with less inhibitory control, and less sophisticated cognitive skills chose riskier routes. Multiple regression analyses showed sex and age, but not inhibitory control, predicted route selection, partially replicating previous research (Barton $\&$ Schwebel, 2007) and accounting for $37 \%$ of the variance in route selections. Next, a hierarchical multiple regression analysis tested the unique contribution of cognitive skills beyond age and sex as predictors of pedestrian route selection. Cognitive factors accounted for an additional $12 \%$ of variance in route selections, significantly improving the model.

Our results support the idea that cognitive developmental factors should be considered when assessing children's potential risk, rather than simply relying on age to determine when children are capable pedestrians.

\section{Reference}

Barton BK and Schwebel DC. The influence of age, gender, and temperament on children's selection of risky pedestrian routes. Journal of Pediatric Psychology 2007;32:343-53. 